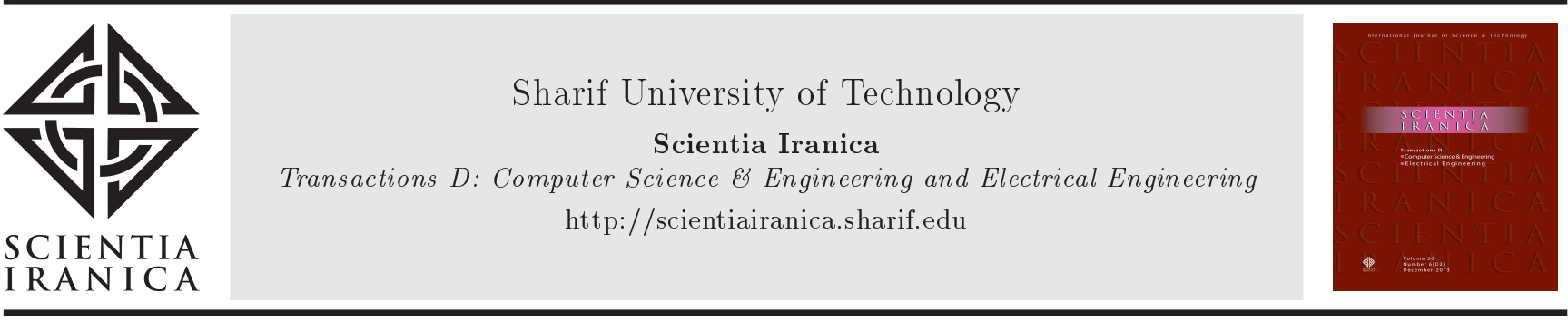

\title{
Developing a toolbox for clinical preliminary breast cancer detection in different views of thermogram images using a set of optimal supervised classifiers
}

\author{
A.E. Lashkari* and M. Firouzmand \\ Department of Bio-Medical Engineering, Institute of Electrical Engineering \& Information Technology, Iranian Research \\ Organization for Science and Technology (IROST), Tehran, Iran.
}

Received 3 December 2015; received in revised form 19 August 2016; accepted 3 September 2016

\author{
KEYWORDS \\ Breast cancer \\ detection; \\ Thermography; \\ Classification; \\ Clinical applications; \\ Ice test; \\ Feature selection; \\ Supervised classifiers.
}

\begin{abstract}
A full automatic technique and a user-friendly toolbox are developed to assist physicians in early clinical detection of breast cancer. The database contains different degrees of thermal images obtained from normal or cancerous mammary tissues of patients with mean age of 42.3 years (SD: \pm 10.50 ), whose sympathetic nervous systems were activated with a cold stimulus on hands. First, ROI was determined using full automatic operation and the quality of image was improved. Then, some features, including statistical, morphological, frequency-domain, histogram, and GLCM, were extracted from segmented right and left breasts. Subsequently, to achieve the best feature space for decreasing complexity and increasing accuracy, feature selectors such as mRMR, SFS, SBS, SFFS, SFBS, and GA were used. Finally, for classification and TH labeling, supervised learning techniques such as AdaBoost, SVM, kNN, NB, and PNN, were applied and compared with each other to find the most suitable one. The experimental results obtained on native database showed the mean accuracy of $88.03 \%$ for 0 -degree images using combination of mRMR and AdaBoost and for combined 3 degrees using combination of GA and AdaBoost. The maximum accuracy obtained from all degrees and their combinations before and after ice test was nearly $100 \%$.
\end{abstract}

(C) 2018 Sharif University of Technology. All rights reserved.

\section{Introduction}

Breast cancer is the most common type of cancer among women. The important key to treat is early detection of it, because, according to many pathological studies, most abnormalities are still benign at primary stages; thus, in recent years, many studies and extensive research have been done for early detection of breast cancer with higher precision and

\footnotetext{
*. Corresponding author. Tel.: +98 9374055672 E-mail address: Lashgari.a@irost.ir (A.E. Lashkari)
}

doi: $10.24200 /$ sci. 2017.4362 accuracy $[1,2]$. There are a number of techniques such as mammography, ultrasound, and MRI for diagnosing the breast cancer. Each of these has well-known advantages and disadvantages. Mammography has been considered as the gold standard for detection of breast cancer, but still suffers from limited sensitivity, specificity, and several other limitations as a screening tool. Ultrasound is used as an adjunctive tool in coordination with mammography in screening women with dense breasts. The limitations of MRI are weak performance in detection of ductal carcinoma in situ, slowness, expensiveness, and inability to show all calcifications [3]. However, all these modalities suffer from one or more common disadvantages related to 
radiation exposure, breast compression, only structural imaging, and breast density [3-5]. In order to resolve the disadvantages of current methods, infrared thermography can be used as a complementary method for detecting breast abnormalities in thermal images. Infrared thermography uses a highly sensitive infrared camera to obtain the image of the temperature distribution in the human body. This imaging technique, due to the use of non-ionizing radiation (passiveness), non-contrast enhanced injection, non-contact, and noninvasiveness, is very valuable in many biological and medical applications in comparison or in combination with other imaging modalities. Moreover, recording temperature distribution patterns in thermography leads to the physiological interpretation of tissues (temperature change based on interaction patterns), which could reveal suspected areas of cancer tissue even when there is no anatomical abnormalities on the tissue surface and it seems quite healthy [6-9]. Also, some research on the temperature dynamic variation of the body surface after cold stimulation of patients has been done that may have advantages in helping physicians, especially in early disease diagnosis. Stimulation of the sympathetic nervous system causes a constriction of the normal blood vessels in the breast. The blood vessels supplying a cancerous tumor will resist constriction or fail to constrict. Consequently, dynamic digital thermal subtraction gives us the possibility of isolating the vessels that supply nutrients to the tumor. This test ultimately raises the suspicion that a cancerous tumor might be present [10]. Thus, this study is an effort to diagnose breast cancer by processing the information obtained from medical infrared imaging. Table 1 shows the related studies done on breast cancer detection using thermography techniques [11-22].

In the present study, due to a lot of attractions of breast thermography, a method with a very noticeable accuracy, sensitivity, and verity has been proposed and a user friendly toolbox is developed to assist physicians in early clinical detection of breast cancer based on different degrees of thermal images. First, the Region Of Interest (ROI) is determined using full automatic operation and the quality of image is improved. Then, some features, including statistical, morphological, frequency-domain, histogram, and Gray Level Co-occurrence Matrix (GLCM), are extracted from segmented right and left breasts. Subsequently, to achieve the best features and increase the accuracy of the proposed method, feature selectors such as minimum Redundancy and Maximum Relevance (mRMR), Sequential Forward Selection (SFS), Sequential Backward Selection (SBS), Sequential Floating Forward Selection (SFFS), Sequential Floating Backward Selection (SFBS), and Genetic Algorithm (GA) have been used. Finally, for classification and $\mathrm{TH}$ labeling procedures, supervised learning techniques such as AdaBoost, Support Vector Machine (SVM), k-Nearest Neighbors (kNN), Naive Bayes (NB), and Probabilistic Neural Network (PNN) are applied and compared with each other to find the most suitable one. The results obtained on native database show the significant performance of the proposed algorithm in comparison with similar studies.

\section{Patients and methods}

In this research, to evaluate the performance of the proposed method, the native database has been used. The thermography images are obtained by Fanavaran Madoon Ghermez (FMG) Co., Ltd [23]. infrared camera (Thermoteknix VisIR 640, Resolution: $480 \times$ $640)$ from the patients with mean age of 42.3 years (SD: \pm 10.50 ), who were suspicious to have breast cancer, referred to Imam Khomeini hospital [24] by their physicians and labeled as $\mathrm{TH}_{1}-\mathrm{TH}_{5}$ depending on the stage of cancer by an oncologist observing all ethical issues. This database contains images with different angles of $0^{\circ}, \pm 45^{\circ}$, and $\pm 90^{\circ}$ before and after ice test (Ice Test: Putting hands in a mixture of ice and water for about $20 \mathrm{~min}$ ) with a total of 10 images for each individual. The total number of people participating until writing this paper was 67 (total 670 images). In this paper, 3 views, i.e. $0^{\circ},+45^{\circ}$, and $+90^{\circ}$, of images after the ice test for all 67 participants have been used and compared with images before the ice test that were obtained before [25].

The camera has been designed in a way that always reduces/eliminates technical errors in the imaging process. For medical applications, a controlled environment helps to increase accuracy. Infrared camera should be placed at certain distance from the patient. Some of the pixels are considered as foreground. Thus, there are parameters that can be completely useful and effective in testing/diagnosis or treatment and instructions that should be observed during data collecting procedures [26]. In the previous paper [25], these parameters and instructions were mentioned. During the experiments, we asked the patients to put their hands on their heads in rest state. Images in five different angles $(0, \pm 45$, and \pm 90$)$ were obtained (before ice test). Then, the patients were asked to put their hands into the mixture of water and ice for about 20 minutes and another images in the five different angles $(0, \pm 45$, and \pm 90$)$ were obtained (after the ice test).

Table 2 shows the distribution of patients whose cancer stages (Standard Level: $\mathrm{TH}$ ) are labeled by the oncologist according to a paper by Gautherine et al. [27] for both right and left breasts separately. The proposed method includes four stages: (1) preprocessing and segmentation, (2) feature extraction, (3) feature selection, and (4) classification and $\mathrm{TH}$ 
Table 1. The previously proposed methods for detecting breast cancer using thermogram images.

\begin{tabular}{ll}
\hline \multicolumn{1}{c}{ Study } & \multicolumn{1}{c}{ Outcome } \\
\hline Williams et al. [11] & $\begin{array}{l}\text { They showed the possibility of detecting breast cancer from increased temperature } \\
\text { at breast tissue acquired by infrared thermography, } \\
\text { (Temperature sensitivity was about } 2{ }^{\circ} \mathrm{C} \text { in the image acquired during a few minutes.) }\end{array}$ \\
\hline & $\begin{array}{l}\text { Clinical trial on } 769 \text { patients was performed for four years; they reported that } \\
\text { infrared thermal imaging was a noninvasive and safe technique, and could be } \\
\text { varisky et al. [12] valuable in determination of the benign or malignant breast } \\
\text { abnormalities combined with mammography }\end{array}$ \\
\hline
\end{tabular}

Arora et al. [13] They showed that the combination of thermotherapy and other diagnostic techniques could and Kennedy et al. [14] improve the accuracy of breast abnormality detection.

A new criterion for breast cancer detection and the method of its realization were proposed
as follows:
1) Surface temperature distribution of healthy breast usually
exhibits a gentle variation, which is background.
2) Localized surface temperature of a carcinomatous breast will increase
on the basis of the above background, which is LTI (Localized Temperature Increases.)
3) The carcinomatous possibility is proportional to the LTI maximum
(amplitude) of the suspicious focus region.
4) The LTI amplitude can be measured through morphological signal processing.

The breast cancer analysis based on thermography was performed using

Schaefer et al. [16] a series of statistical features extracted from the thermograms, quantifying the bilateral differences between left and right breast areas, coupled with a fuzzy rule-based classification system for diagnosis.

Texture features were extracted from co-occurrence matrix and run length

Acharya et al. [17] matrix. Subsequently, these features were fed to the Support Vector Machine (SVM) classifier for automatic classification of normal and malignant breast conditions.

Wiener filter was used to eliminate the noise. By using histogram equalization,

Satoto et al. [18] region growing method. Then, statistical features such as mean, standard image contrast improved. The last step of the preprocessing procedure used deviation, entropy, skewness, and kurtosis of the images were extracted and, finally, the fuzzy algorithm was used for classification.

Background and additional areas of the image were removed first. Breast boundaries were extracted using "canny" edge detection and gradient operator was used to determine the boundary curves of the left and right breasts. Lower bounds of breast were determined using two

Kapoor et al. [19,20] elliptical curves and the area under the curve was eliminated. Then, a separator for separating the right and left breasts was used. In the feature extraction stage, the features of skewness, kurtosis, and entropy as well as features based on co-occurrence matrix such as energy, homogeneity, and correlation were extracted. Finally, the MLP neural network was used for classification of features.

Information such as the temperature difference between the left and right breasts, hot spots in the breast, the temperature difference between the hot spots, and the center of the hot zone as well as features based on the

Nicandro et al. [21] histogram, patient age, etc. were used to determine the suspected areas. To evaluate the performance, Naive Bayes classifier, hill climbing, iterative hill climbing, artificial neural networks, and the ID3 and C4.5 decision trees were used. The iterative hill climbing algorithm had the best performance with the accuracy of $76.12 \%$ among all classifiers. 
Table 1. The previously proposed methods for detecting breast cancer using thermogram images (continued).

\begin{tabular}{cl}
\hline Study & \multicolumn{1}{c}{ Outcome } \\
\hline & First, using CLAHF (Contrast Limited Adaptive Histogram Equalization), \\
& the quality of thermal images was improved and the noise was eliminated \\
& using a non-linear filter. To achieve strong edges, Gaussian filter with \\
Dinsha and & weighting coefficients corresponding to pixel intensities was developed. \\
Manikandaprabu [2] & Segmentation was performed using both $k$-means and FCM (Fuzzy C Means) algorithms \\
& and features based on co-occurrence matrix separately. Finally, \\
& SVM (Support Vector Machine) and Bayesian classifiers were used in feature space. \\
\hline
\end{tabular}

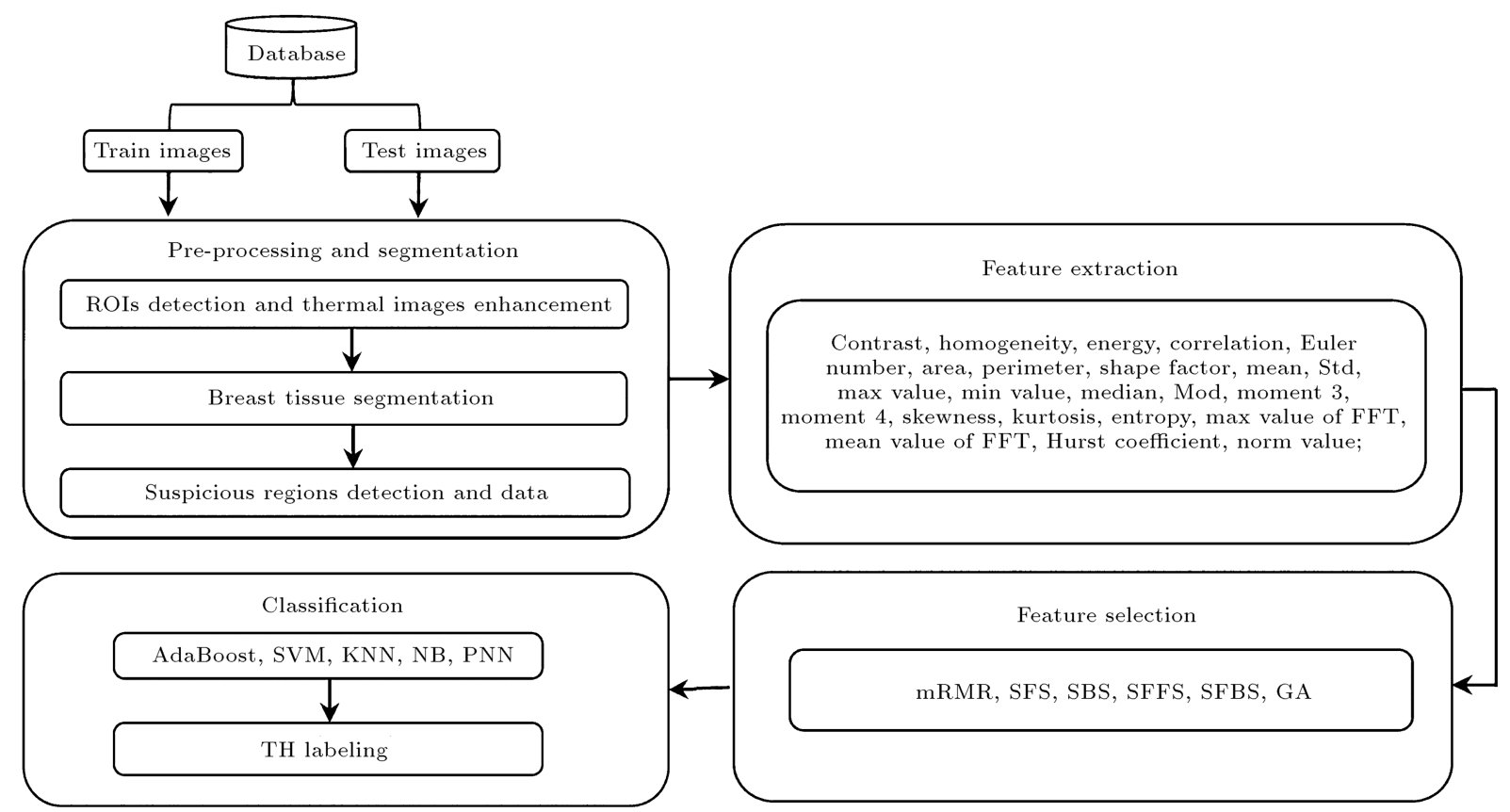

Figure 1. Proposed system architecture.

Table 2. The distribution of standard level labeled by oncologist for both right and left breasts separately.

\begin{tabular}{cccccc}
\hline TH & $\mathbf{1}$ & $\mathbf{2}$ & $\mathbf{3}$ & $\mathbf{4}$ & $\mathbf{5}$ \\
\hline Right breast & 24 & 22 & 12 & 4 & 5 \\
Left breast & 6 & 28 & 14 & 13 & 6
\end{tabular}

labeling. Figure 1 shows structure of the presented method. Finally, a toolbox is developed for clinical applications (Figures 2 and 3 ).

\subsection{Pre-processing and segmentation}

Pre-processing, especially in data driven studies, is a very important stage. To distinguish between normal and abnormal tissues, pre-processing and segmentation are performed in three stages:

1. Detection of Regions Of Interest $\left(\mathrm{ROI}_{s}\right)$ and enhancement of thermal images;

\section{Breast tissue segmentation;}

3. Detection of suspicious regions and image matrix normalization.

\subsection{Feature extraction}

In the feature extraction stage, some information from right and left breast images should be extracted to distinguish between normal and abnormal tissues, correctly. The extracted features are same as those in the previous paper [25] and are based on statistics such as median, mod, skewness, kurtosis, central moment, and entropy; histograms such as mean, standard deviation, max-value, min-value, and norm; GLCMs such as contrast, homogeneity, energy, and correlation; morphology of suspicious regions such as Euler number, area, perimeter, shape factor, and Hurst coefficient; and frequency domain such as max value of fast Fourier transform and mean value of fast Fourier transform. 


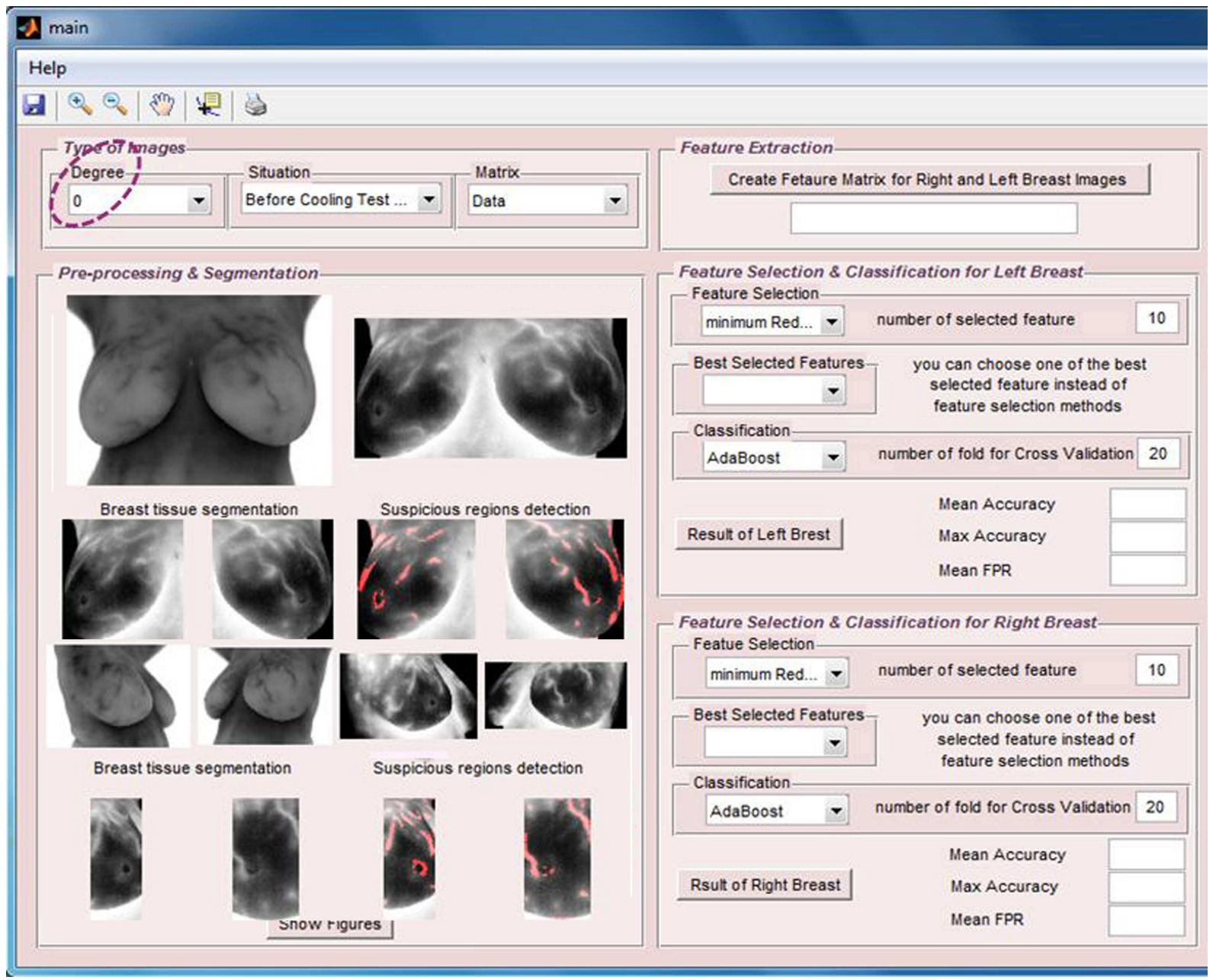

Figure 2. 45-degree view of the clinical toolbox.

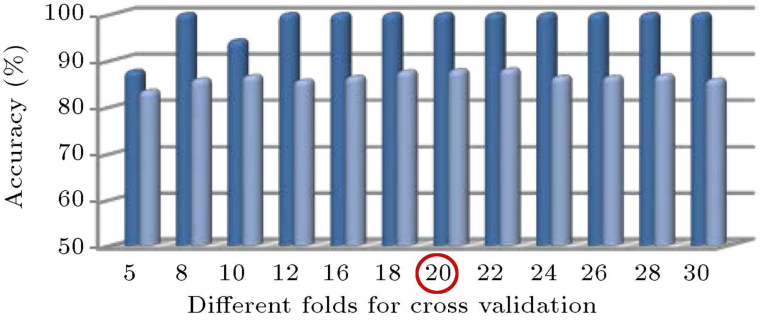

(a)

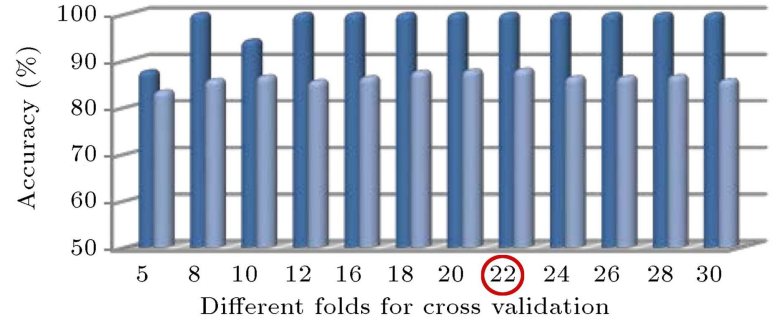

(b)

Figure 3. The accuracies obtained for a number of different folds for (a) left breast images, and (b) right breast images. Dark blue: max accuracy, light blue: mean accuracy.

\subsection{Feature selection}

Various information causes high dimensionality of the obtained feature matrix that reduces accuracy and increases computation burden. Therefore, to attain more accurate selection and further reduction in the number of extracted features, different feature selection methods are used. In this paper, the minimalRedundancy and Maximal-Relevance (mRMR) [28], Sequential Forward Selection (SFS) [29], Sequential Backward Selection (SBS) [29], Sequential Floating
Forward Selection (SFFS) [30], Sequential Floating Backward Selection (SFBS) [30], and Genetic Algorithm (GA) [31] are applied and, then, compared with each other.

\subsection{Classification and TH labeling}

Classification is the final stage in the proposed approach. Thus, the selected feature matrix is fed into the classification algorithm to detect TH. TH is a standard measure for thermovasculars analysis of 
the breast that was proposed in 1980s. Physicians classify thermal images into five categories based on the combined vascular and temperature patterns across the two breasts as: $\mathrm{TH}_{1}$ : normal nonvascular, $\mathrm{TH}_{2}$ : normal vascular, $\mathrm{TH}_{3}$ : equivocal, $\mathrm{TH}_{4}$ : abnormal, $\mathrm{TH}_{5}$ : Severely abnormal [27]. In this research, to show the performance of the proposed method, different classification algorithms have been applied and their results have been evaluated and compared with each other to obtain the best results. These algorithms are AdaBoost, [32,33] Support Vector Machine (SVM) [34], kNearest Neighbors (k-NN) [35], Naïve Bayes (NB) [35], and Probabilistic Neural Network (PNN) [36]. The description of each method is accessible in [25].

\subsection{Developing clinical user friendly toolbox}

As showed in Figures 2 and 3, all the stages are implemented and developed in compatible User Friendly Toolbox to assist the physicians in using the proposed algorithm for all clinical breast cancer detection applications.

\section{Results}

In this research, to evaluate the performance of the proposed method, the native database with 67 thermography images from 10 people (total 670 images) has been used. The thermography images were obtained by Fanavaran Madoon Ghermez (FMG) Co., Ltd [23] with infrared camera (Thermoteknix VisIR 640, Resolution: $480 \times 640)$ in Imam Khomeini Hospital [24] by their physician and labeled as $\mathrm{TH}_{1}-\mathrm{TH}_{5}$ depending on the stage of cancer by an oncologist observing all ethical issues. This database contained images with different angles of $0^{\circ}, \pm 45^{\circ}$, and $\pm 90^{\circ}$ before and after ice test. Before the ice test, 3 views of images, i.e. $0^{\circ}$, $+45^{\circ}$, and $+90^{\circ}$, were used and compared for all 67 participants.

In the proposed approach, after determining ROIs and enhancing thermal images, right and left breast tissues were separated by edge detection operators. To detect suspicious regions in each breast, "Erosion" morphological was used. Also, to evaluate the suspicious regions in thermogram images, different degrees of breast images were used based upon which performance of the proposed algorithm was validated [25]. Then, the mentioned features were extracted from right and left breast images. To obtain optimal features, different feature selection methods were applied. Finally, the selected feature matrix was fed to different classifiers for determining TH; AdaBoost showed the best result among the other classifiers. Experiments show that the best result was obtained when $k$, the number of neighbors in k-NN classifier, was equal to 3 ; the smoothing parameter in PNN classifier was equal to 0.35; Gaussian kernel of degree 6 was used for the
SVM classifier; and value of parameter $C$ was considered equal to 1 . To evaluate the performance of the proposed technique, different folds were studied. According to the diagrams in Figure 3(a) and (b), the best result has been obtained with 20 and 22 fold cross validations for the left and right breast images, respectively. In these figure, the mean and maximum accuracy obtained for different folds can be seen [25]. In addition, to show the performance of the provided method, some measures such as sensitivity, specificity, Area Under Curve (AUC), Equal Error Rate (EER), precision, recall, F-measure, and FPR were also calculated.

\subsection{Evaluation of extracted features based on feature selectors}

To obtain optimal feature space, 6 feature selection methods, namely, mRMR, SFS, SBS, SFFS, SFBS, and GA are used in the two situations before and after ice test. The feature space obtained before ice test has been studied in the previous paper [25] and after ice test is studied in this paper. In the proposed algorithm, each feature selected by several feature selection methods for several times on the right and left breasts can be introduced as an effective feature. To better understand the approach, it is necessary to note that:

- Each of the feature selection methods is evaluated with different classifiers;

- In SFS, SBS, SFFS, and SFBS, the features are selected for one time; then, they are evaluated by different classifiers;

- In mRMR and GA, the optimal number of features should be obtained; Thus, the numbers and types of the selected features by these 2 methods will be different by applying different classifiers. Also, as the outcome of the combination of mRMR and GA methods with 5 classifiers, each feature may be selected once or more. The obtained values show that a feature may be evaluated by different classifiers as good feature and it is able to increase the accuracy of the algorithms;

- Features could be suitable if they are selected by at least 3 of the feature selection methods in left or right breast images;

- Features could be good if they are selected by at least 2 of the feature selection methods, but the mRMR and GA methods have selected them several times;

- Features are not very good or very bad if they are selected by at least 2 of the feature selection methods and mRMR and GA methods have selected them few times; 
- Features are not very good (bad) if they are selected by only one of the feature selection methods and one of the mRMR and GA;

- Features are not suitable if they are not selected or are selected by only one method.

The effective features before ice test [25] were Contrast, Euler number, area, mean, central moment 4, and skewness for 0_degree images; contrast, perimeter, shape_factor, max_value, min_value, mod, skewness, kurtosis, entropy, mean value of FFT, and norm value for 45_degree images; and area, shapefactor, standard deviation, central moment 3 , central moment4, kurtosis, max value of FFT, mean value of FFT, Hurst coefficient, and norm value for 90_degree images. Moreover, the effective features before ice test for the combination of the 3 degrees were Euler number, area, perimeter, central moment 3 , central moment 4 , skewness, kurtosis, entropy, max value of FFT, Hurst coefficient, and norm value.

\subsection{Evaluation of different feature selection methods for right and left breasts in different degrees}

In the proposed method, to obtain optimal features, different feature selection methods have been used. The probability of selecting each feature is specified by one of the feature selection methods after ice test situation. The results for the before-ice-test situation have been reported before [25]. As before, the numbers and types of the selected features in mRMR and GA methods will be different by applying different classifiers. Thus, in this experiment, to attain the highest accuracy of the AdaBoost classifier, it is used in combination with $\mathrm{mRMR}$ and GA to select the features.

\subsection{Performance assessment of the proposed method}

In this section, the performance of the proposed method has been evaluated with combinations of different feature selection methods and classifiers. 5 classification methods, namely, AdaBoost, SVM, KNN, NB, and PNN, combined with 6 feature selection methods, namely, mRMR, SFS, SBS, SFFS, SFBS, and GA, have been used. The results are expressed by using boxwhisker plots in Figures 4-6 for 0, 45, and 90 degrees, respectively and in Figure 7 for the combination of 0 , 45, and 90 degrees before and after ice test. Horizontal axis shows different classifiers and vertical axis shows measures for evaluating the performance of the method. Box-plot can also show average and median. On each box, the horizontal line denotes median, the circle denotes mean, the horizontal lines outside each box identify the upper and lower whiskers, and points denote the outliers. The dotted line in each figure shows the highest mean accuracy for combination of one feature selection method with one classification method among the other combinations in left and right breast images. The best combination obtained is noted in these figures. As can be seen in Figures 4-6, AdaBoost has the best performance compared with the other classifiers. Thus, the combinations of AdaBoost with 6 feature selection methods have been studied in ROC curves in Figures 8-11 to evaluate different feature selectors. Moreover, AdaBoost without feature selector has been shown in these ROC curves. The best combination obtained is shown in the box-whisker plots, which is also evident in ROC curves, and the performance proximity of the feature selectors can also be seen in ROC curves. It should be noted that the placement of some curves might be different from their situations in box-whisker plots, because two-class classification has been used to plot ROC curves.

\subsection{Evaluation of the proposed method in different degrees of breast images before and after ice test}

As mentioned in the previous sections, to evaluate the proposed algorithm, different degrees of breast images are considered in both situations before and after ice test. Now, the best accuracies obtained by the best combinations in different conditions have been studied in this section and the obtained results are compared with each other in Table 3 . The following results can be obtained from Table 3 .

\subsubsection{Comparing results based on different degrees}

- The arrangement of accuracy obtained for the right breast images before ice test:

combination of $0^{\circ} \& 45^{\circ} \& 90^{\circ}>0^{\circ}>90^{\circ}>45^{\circ}$.

- The arrangement of accuracy obtained for the left breast images before ice test: $0^{\circ}>45^{\circ}>90^{\circ}>$ combination of $0^{\circ} \& 45^{\circ} \& 90^{\circ}$.

- The arrangement of accuracy obtained for the right breast images after ice test: $0^{\circ}>$ combination of $0^{\circ} \& 45^{\circ} \& 90^{\circ}>45^{\circ}>90^{\circ}$.

- The arrangement of accuracy obtained for the left breast images after ice test: combination of $0^{\circ} \& 45^{\circ} \& 90^{\circ}>90^{\circ}>45^{\circ}>0^{\circ}$.

\subsubsection{Comparing results before and after ice test situation}

- The arrangement of accuracy obtained for the right breast images in different degrees:

- Before ice test Right $\left(0^{\circ}, 45^{\circ}\right)<$ after ice test $_{\text {Right }}$ $\left(0^{\circ}, 45^{\circ}\right)$;

- Before ice test Right $\left(90^{\circ}\right)>$ after ice test Right $_{\text {}}$ $\left(90^{\circ}\right)$;

- Before ice test Right (combination of $0^{\circ} \& 45^{\circ} \&$ $\left.90^{\circ}\right)$ 


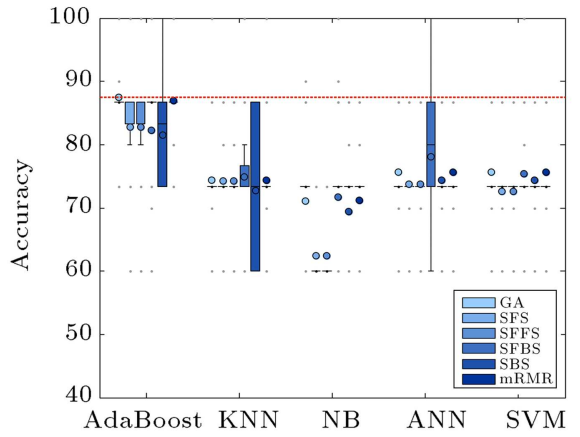

(a)

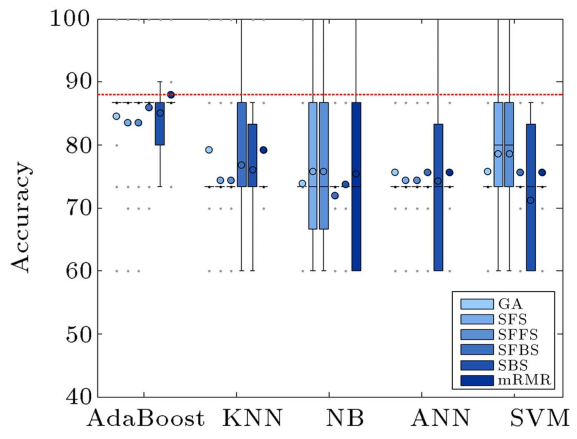

(c)

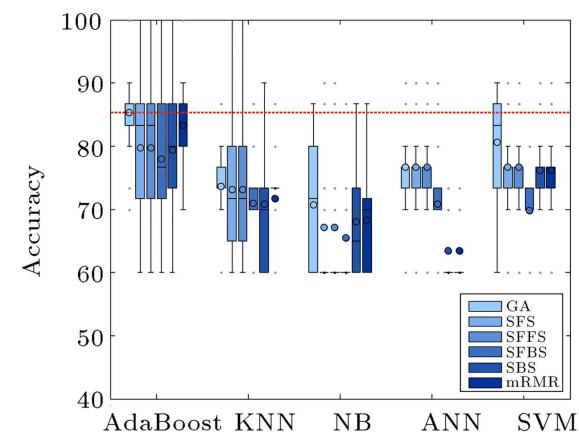

(b)

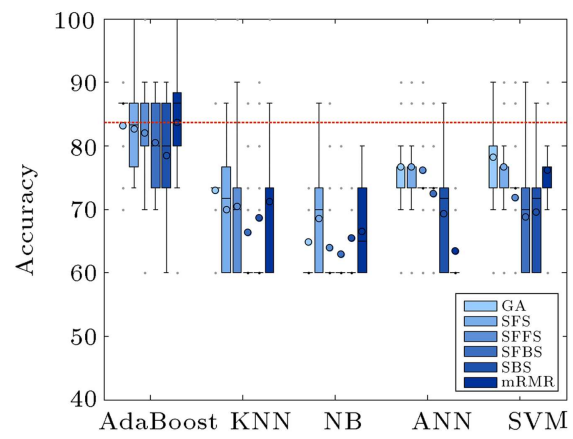

(d)

Figure 4. Accuracy of the proposed system corresponding to different feature selectors and classifiers on 0-degree breast images: (a) Right breast images before ice test (best combination: GA and AdaBoost), (b) left breast images before ice test (best combination: GA and AdaBoost), (c) right breast images after ice test (best combination: mRMR and AdaBoost), and (d) left breast images after ice test (best combination: mRMR and AdaBoost).

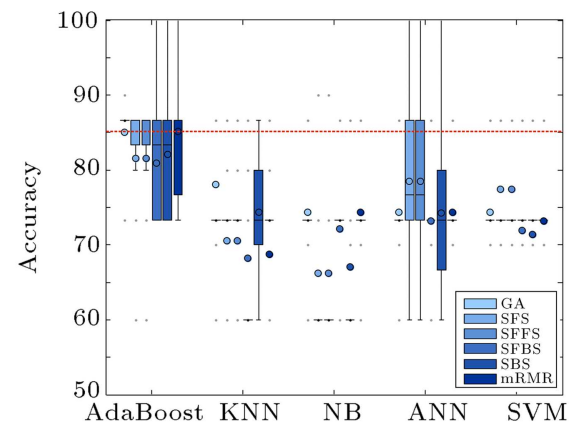

(a)

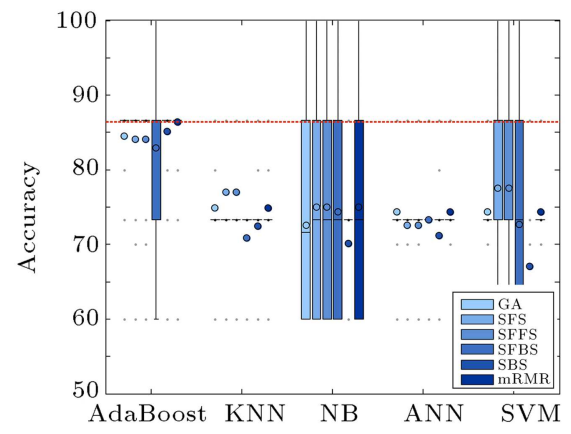

(c)

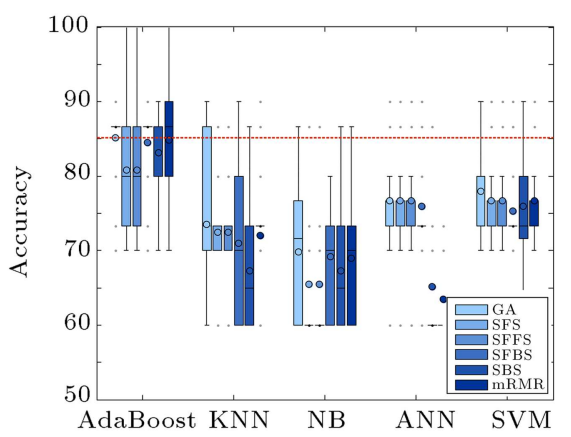

(b)

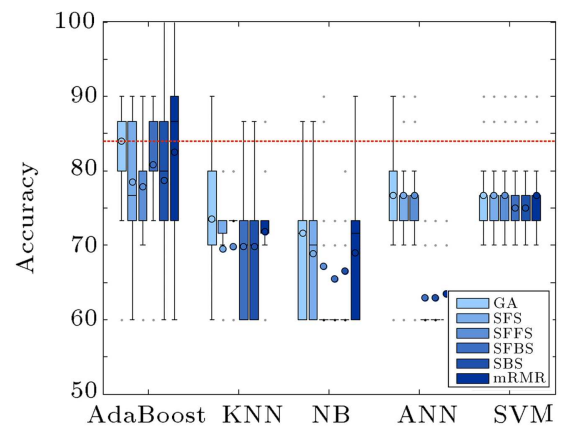

(d)

Figure 5. Accuracy of the proposed system corresponding to different feature selectors and classifiers on 45-degree breast images: (a) Right breast images before ice test (best combination: mRMR and AdaBoost), (b) left breast images before ice test (best combination: GA and AdaBoost), (c) right breast images after ice test (best combination: mRMR and AdaBoost), and (d) left breast images after ice test (best combination: GA and AdaBoost). 


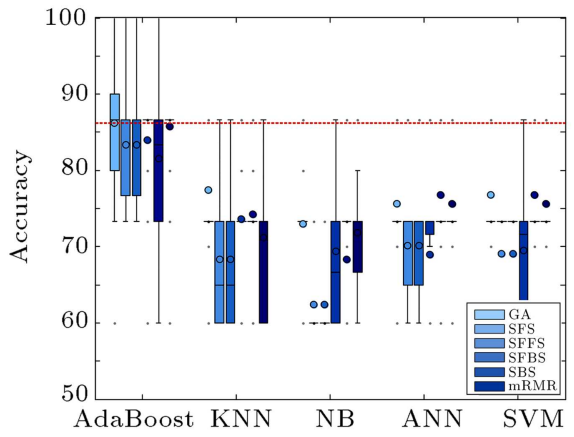

(a)

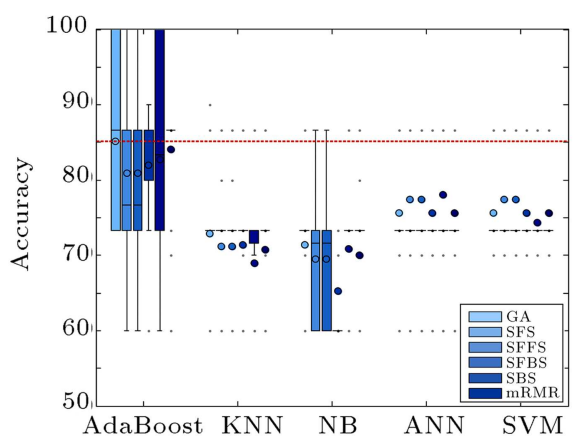

(c)

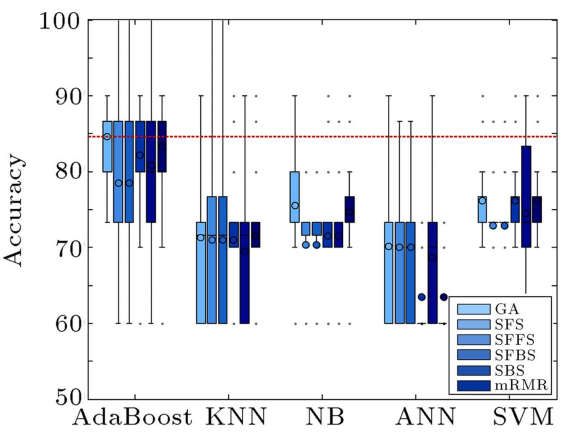

(b)

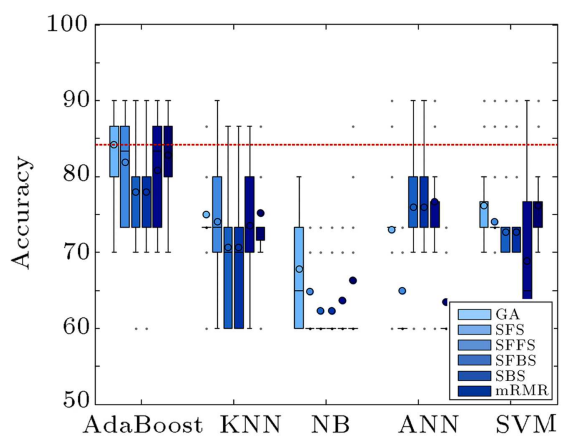

(d)

Figure 6. Accuracy of the proposed system corresponding to different feature selectors and classifiers on 90-degree breast images: (a) Right breast images before ice test (best combination: GA and AdaBoost), (b) left breast images before ice test (best combination: GA and AdaBoost), (c) right breast images after ice test (best combination: GA and AdaBoost), and (d) left breast images after ice test (best combination: GA and AdaBoost).

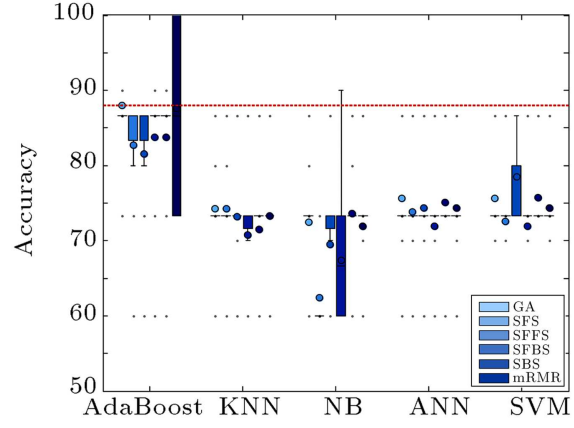

(a)

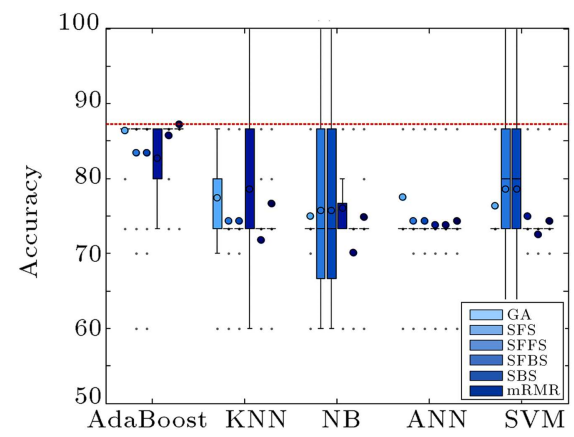

(c)

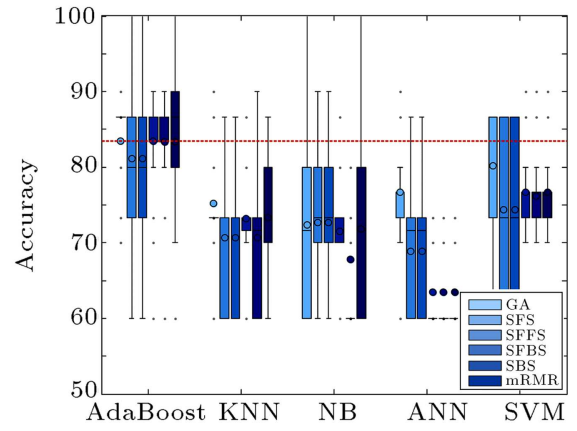

(b)

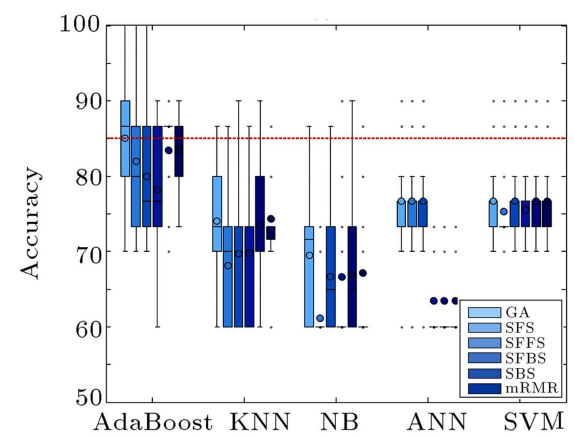

(d)

Figure 7. Accuracy of the proposed system corresponding to different feature selectors and classifiers on the combination of 0-, 45-, and 90-degree breast images: (a) Right breast images before ice test (best combination, GA and AdaBoost), (b) left breast images before ice test (best combination, GA and AdaBoost), (c) right breast images after ice test (best combination: mRMR and AdaBoost), and (d) left breast images after ice test (best combination: GA and AdaBoost). 


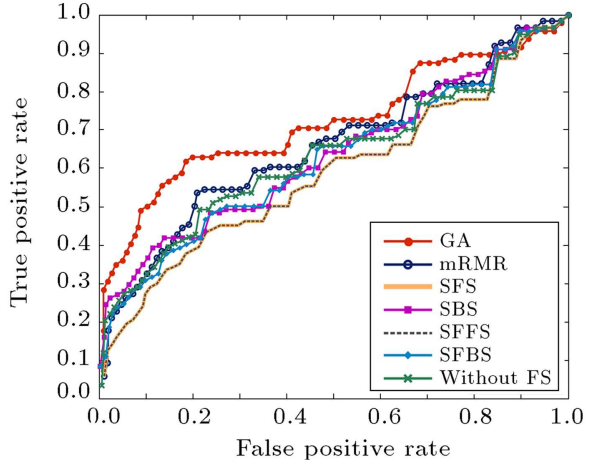

(a)

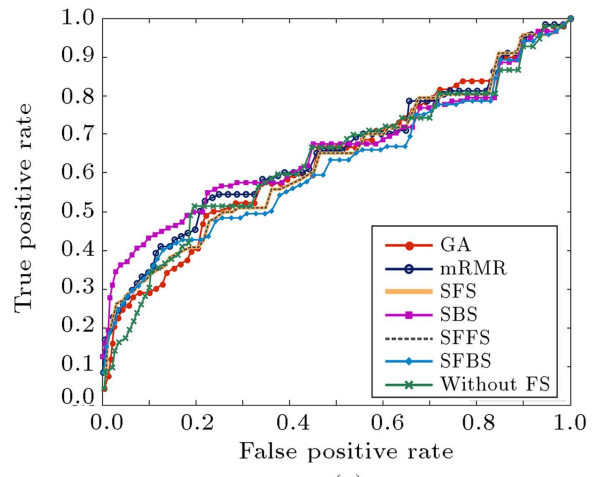

(c)

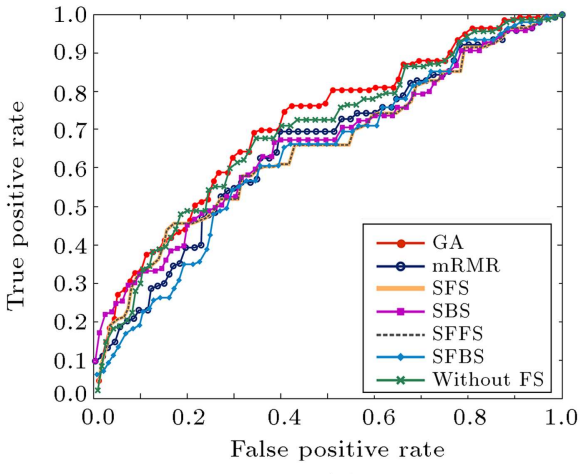

(b)

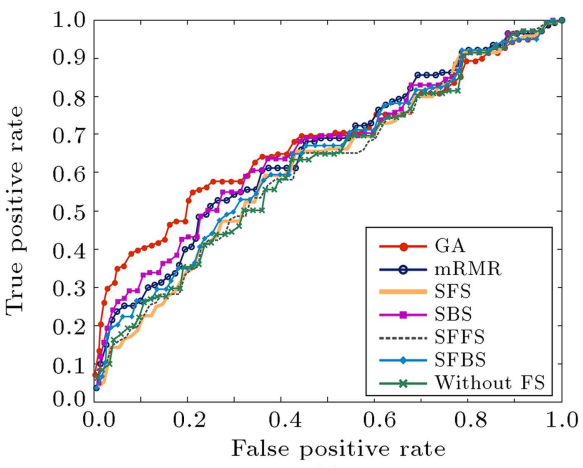

(d)

Figure 8. ROC curves for comparison of different feature selection methods in 0-degree breast images: (a) Comparing results for right breast images before ice test, (b) comparing results for left breast images before ice test, (c) comparing results for right breast images after ice test, and (d) comparing results for left breast images after ice test.

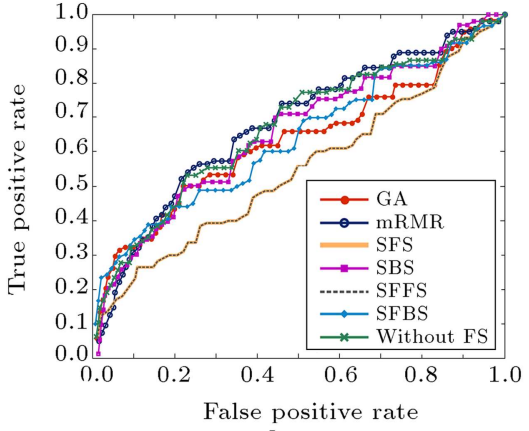

(a)

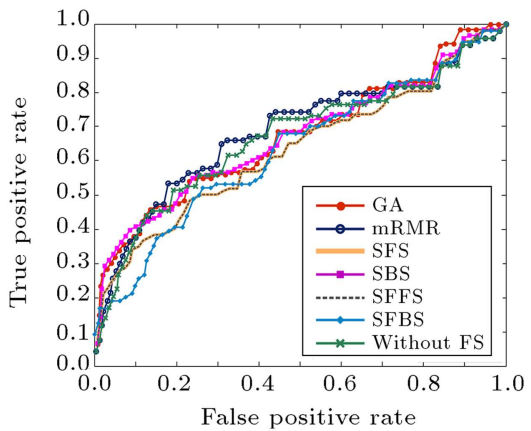

(c)

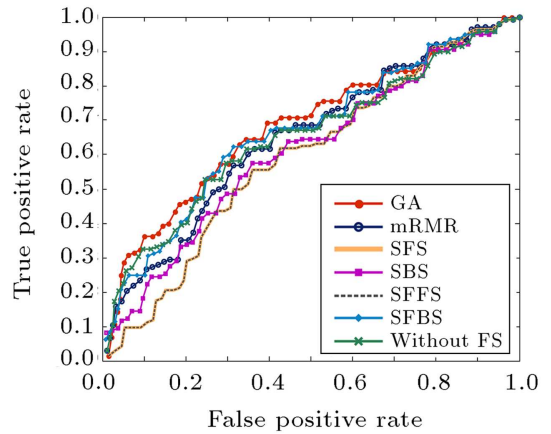

(b)

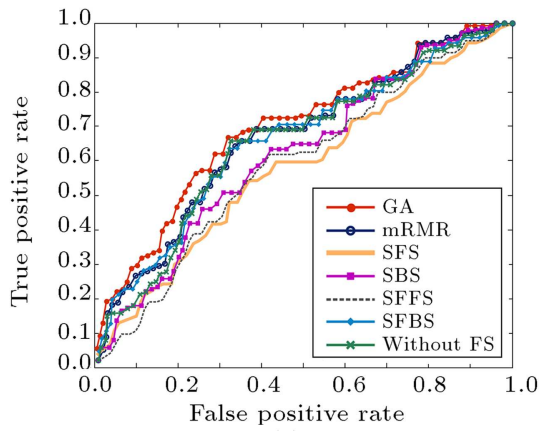

(d)

Figure 9. ROC curves for comparison of different feature selection methods in 45-degree breast images: (a) Comparing results for right breast images before ice test, (b) comparing results for left breast images before ice test, (c) comparing results for right breast images after ice test, and (d) comparing results for left breast images after ice test. 


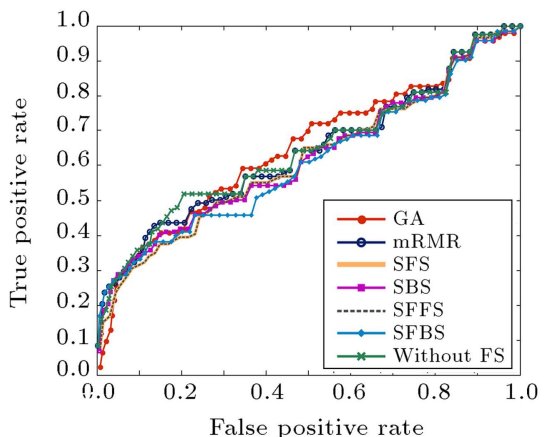

(a)

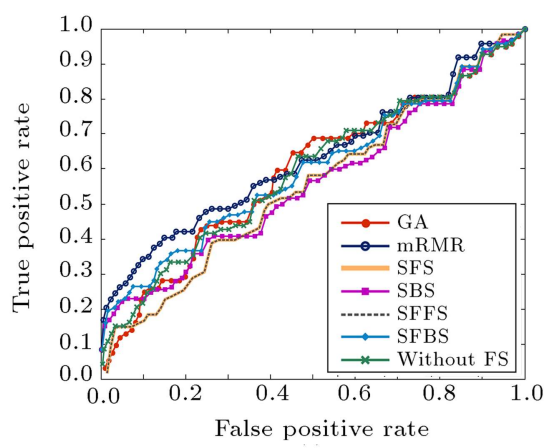

(c)

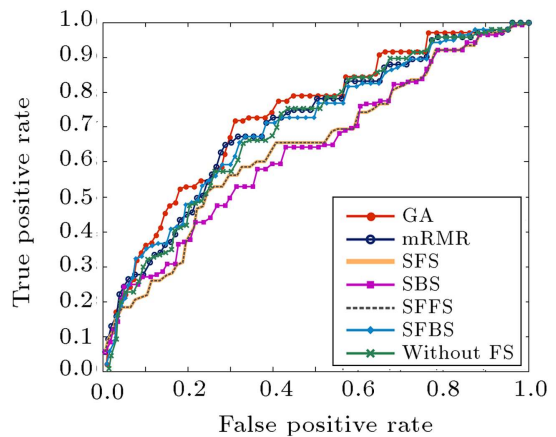

(b)

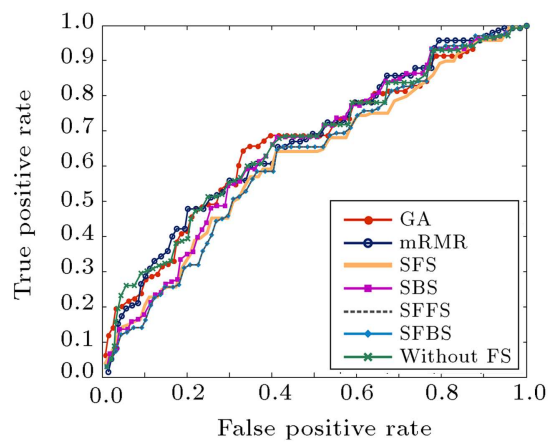

(d)

Figure 10. ROC curves for comparison of different feature selection methods in 90-degree breast images: (a) Comparing results for right breast images before ice test, (b) comparing results for left breast images before ice test, (c) comparing results for right breast images after ice test, and (d) comparing results for left breast images after ice test.

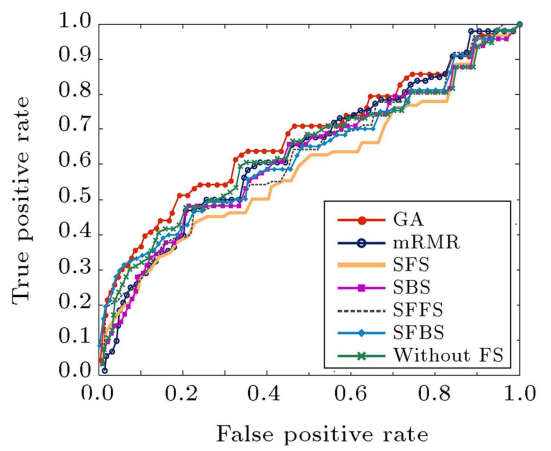

(a)

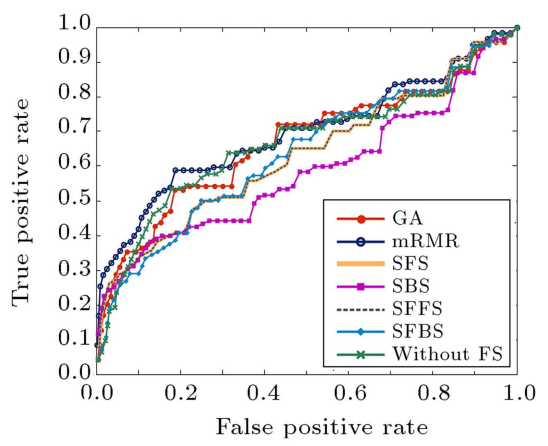

(c)

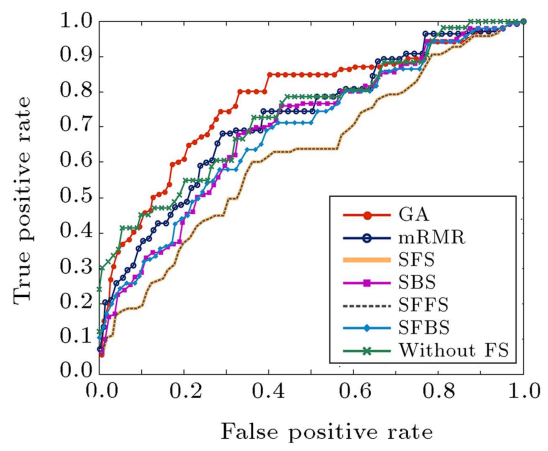

(b)

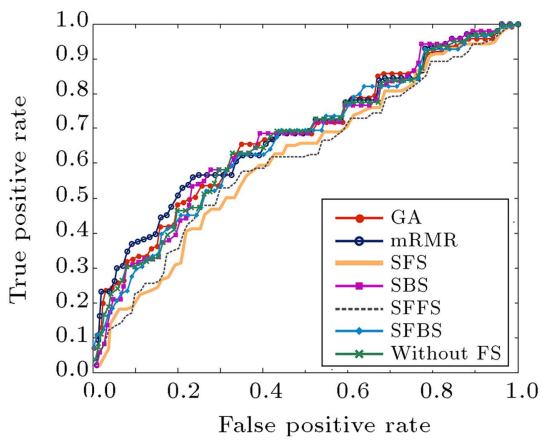

(d)

Figure 11. ROC curves for comparison of different feature selection methods in the combination of 0-, 45-, and 90-degree breast images: (a) Comparing results for right breast images before ice test, (b) comparing results for left breast images before ice test, (c) comparing results for right breast images after ice test, and (d) comparing results for left breast images after ice test. 
Table 3. Evaluation of the best combinations obtained for the right and left breast images in different degrees before and after ice test.

\begin{tabular}{|c|c|c|c|c|c|c|c|c|c|c|}
\hline & Degree & $\begin{array}{c}\text { The best } \\
\text { combinations }\end{array}$ & $\begin{array}{c}\text { Mean } \\
\text { accuracy }\end{array}$ & $\begin{array}{c}\text { Mean }^{a} \\
\text { sens. }\end{array}$ & $\begin{array}{c}\text { Mean }^{b} \\
\text { spec. }\end{array}$ & $\begin{array}{l}\text { Men } \\
\text { AUC }\end{array}$ & $\begin{array}{l}\text { Mean } \\
\text { EER }\end{array}$ & $\begin{array}{c}\text { Mean } \\
\text { F-Measure }\end{array}$ & $\begin{array}{c}\text { Max } \\
\text { accuracy }\end{array}$ & $\begin{array}{l}\text { Mean } \\
\text { FPR }\end{array}$ \\
\hline \multirow{8}{*}{ 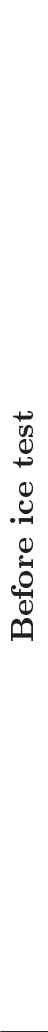 } & & $\begin{array}{c}\text { AdaBoost }+ \\
\text { GA on the left breast }\end{array}$ & $85.33 \%$ & 0.6333 & 0.9083 & $77.08 \%$ & 0.2292 & $63.33 \%$ & $100 \%$ & 0.0917 \\
\hline & 0 & $\begin{array}{c}\text { AdaBoost }+ \\
\text { GA on the right breast }\end{array}$ & $87.42 \%$ & 0.6856 & 0.9214 & $80.35 \%$ & 0.1965 & $68.56 \%$ & $100 \%$ & 0.0786 \\
\hline & & $\begin{array}{c}\text { AdaBoost }+ \\
\text { GA on the left breast }\end{array}$ & $85.17 \%$ & 0.6292 & 0.9073 & $76.82 \%$ & 0.2317 & $62.91 \%$ & $100 \%$ & 0.0927 \\
\hline & 45 & $\begin{array}{c}\text { AdaBoost }+ \\
\text { mRMR on the right breast }\end{array}$ & $87.42 \%$ & 0.6856 & 0.9214 & $80.35 \%$ & 0.1965 & $68.56 \%$ & $100 \%$ & 0.0786 \\
\hline & & $\begin{array}{c}\text { AdaBoost }+ \\
\text { GA on the left breast }\end{array}$ & $84.67 \%$ & 0.6167 & 0.9042 & $76.04 \%$ & 0.2395 & $61.66 \%$ & $100 \%$ & 0.0958 \\
\hline & 90 & $\begin{array}{c}\text { AdaBoost }+ \\
\text { mRMR on the right breast }\end{array}$ & $86.21 \%$ & 0.6553 & 0.9138 & $78.45 \%$ & 0.2154 & $65.53 \%$ & $100 \%$ & 0.0862 \\
\hline & $\begin{array}{l}8 \\
8\end{array}$ & $\begin{array}{c}\text { AdaBoost }+ \\
\text { GA on the left breast }\end{array}$ & $83.50 \%$ & 0.5875 & 0.8969 & $74.21 \%$ & 0.2578 & $58.75 \%$ & $100 \%$ & 0.1031 \\
\hline & $\begin{array}{l}\underset{10}{+1} \\
2 \\
0\end{array}$ & $\begin{array}{c}\text { AdaBoost }+ \\
\text { mRMR on the right breast }\end{array}$ & $88.03 \%$ & 0.7008 & 0.9252 & $81.29 \%$ & 0.1870 & $70.07 \%$ & $100 \%$ & 0.0748 \\
\hline \multirow{8}{*}{ 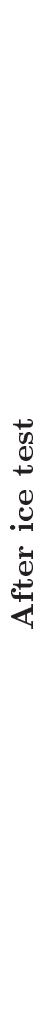 } & & $\begin{array}{c}\text { AdaBoost }+ \\
\text { GA on the left breast }\end{array}$ & $83.67 \%$ & 0.5916 & 0.8979 & $74.47 \%$ & 0.2552 & $59.16 \%$ & $100 \%$ & 0.1021 \\
\hline & 0 & $\begin{array}{c}\text { AdaBoost }+ \\
\text { GA on the right breast }\end{array}$ & $88.03 \%$ & 0.7007 & 0.9251 & $81.29 \%$ & 0.1870 & $70.07 \%$ & $100 \%$ & 0.0748 \\
\hline & & $\begin{array}{c}\text { AdaBoost }+ \\
\text { GA on the left breast }\end{array}$ & $84 \%$ & 0.60 & 0.90 & $75 \%$ & 0.2500 & $60 \%$ & $100 \%$ & 0.10 \\
\hline & 45 & $\begin{array}{c}\text { AdaBoost }+ \\
\text { mRMR on the right breast }\end{array}$ & $86.36 \%$ & 0.6591 & 0.9148 & $78.69 \%$ & 0.2131 & $65.90 \%$ & $100 \%$ & 0.0852 \\
\hline & \multirow[b]{2}{*}{90} & $\begin{array}{c}\text { AdaBoost }+ \\
\text { GA on the left breast }\end{array}$ & $84.17 \%$ & 0.6042 & 0.9010 & $75.26 \%$ & 0.2473 & $60.41 \%$ & $100 \%$ & 0.0990 \\
\hline & & $\begin{array}{c}\text { AdaBoost }+ \\
\text { mRMR on the right breast }\end{array}$ & $85.15 \%$ & 0.6288 & 0.9072 & $76.79 \%$ & 0.2320 & $62.87 \%$ & $100 \%$ & 0.0928 \\
\hline & $\begin{array}{l}8 \\
8\end{array}$ & $\begin{array}{c}\text { AdaBoost }+ \\
\text { GA on the left breast }\end{array}$ & $85 \%$ & 0.6250 & 0.9063 & $76.56 \%$ & 0.2343 & $62.50 \%$ & $100 \%$ & 0.0938 \\
\hline & $\begin{array}{l}\stackrel{2}{+} \\
\infty \\
0\end{array}$ & $\begin{array}{c}\text { AdaBoost }+ \\
\text { mRMR on the right breast }\end{array}$ & $87.27 \%$ & 0.6818 & 0.9205 & $80.11 \%$ & 0.1988 & $68.18 \%$ & $100 \%$ & 0.0795 \\
\hline
\end{tabular}

${ }^{a}$ Mean sens.: Mean sensitivity;

b Mean speci.: Mean specificity. 
- After ice test $t_{\text {Right }}\left(\right.$ combination of $\left.0^{\circ} \& 45^{\circ} \& 90^{\circ}\right)$.

- The arrangement of accuracy obtained for the left breast images in different degrees:

- Before ice test ${ }_{\text {Left }}\left(0^{\circ}, 45^{\circ}, 90^{\circ}\right)<$ after ice test $_{\text {Left }}$ $\left(0^{\circ}, 45^{\circ}, 90^{\circ}\right)$;

- Before ice test ${ }_{\text {Left }}\left(\right.$ combination of $\left.0^{\circ} \& 45^{\circ} \& 90^{\circ}\right)$;

- After ice test $t_{\text {Left }}\left(\right.$ combination of $\left.0^{\circ} \& 45^{\circ} \& 90^{\circ}\right)$;

In these experiments, we expected the accuracy of the proposed method to increase in the combination of 3 degrees of 0,45 , and 90 after ice test, but we witnessed differences in the obtained results. Thus, according to the results mentioned above, the following reasons can be suggested to justify the difference in accuracy of different degrees before and after ice test in the proposed method.

- Challenges of existing the mass or lesion in breast: For example, it is possible that the placement of the mass in the image with 0 degree is better than that in images with other degrees and, consequently, the extracted features will be more suitable. These suitable features may not be suitable and effective in combination with other extracted features from images with 45 and 90 degrees and reduce the accuracy of the proposed method.

- The segmentation fault: For example, it is possible that segmentation of the images with 0 degree is done correctly, but, due to problems in the segmentation of images with 45 and 90 degrees, their segmentation is done slightly incorrectly and the accuracy of the proposed method is decreased. Thus, the incorrect segmentation affects the results of the combination of 3 degrees and the accuracy of the proposed algorithm will be reduced.

- Unstable environment: These experiments are very sensitive to the environment; therefore, all conditions, such as the room temperature, the necessary time for doing experiments on the patients, waiting time for cold stimulation of patients, and the time for putting the patients' hands in ice should be stable.

- Non-uniform temperature distribution: After cold stimulation, because the cold is entered into the breast by the patients' hands, temperature distribution is not uniform in all areas of the breast and the tissue of the breast will not be cold equally. Also, due to distribution of temperature from hand to breast, temperature distribution in images of breast with different degrees will be variable and various accuracy will be obtained.

- Unique temperature of each person: Due to unique temperature of each person, it may not be possible to consider uniform condition for all persons, even if the waiting time for cold stimulation of patients is controlled.

- Change in contrast of breast images: The contrast of images changes by applying the cold stimulation and, as a result, the accuracy of the system may be reduced.

Therefore, due to the existing problems in ice test and little differences in accuracy for different situations, cold stimulation can be omitted. However, this experiment can be used for observing the temperature diagram and thermal changes of everybody before and after ice test and for partly realizing the presence or absence of masses, same as the recent study [10].

\section{Conclusion}

In the proposed method, an imaging technique based on thermography was used to detect early changes occurring in the breast tissue and cancer cells. In this paper, an automatic method was developed for breast cancer detection based on thermography to detect suspicious areas and label the related TH. To obtain the five categories, i.e. $\mathrm{TH}_{1}-\mathrm{TH}_{5}$, four main stages in 3 degrees of 0,45 , and 90 were implemented with or without considering ice test: pre-processing and segmentation, feature extraction, feature selection, and classification. Pre-processing and segmentation stage included detection of region of interest and enhancement of thermal images, segmentation of breast tissues, detection of suspicious areas, and normalization of the image matrix. Some features with different types were extracted from right and left breast images and the effective features were selected by different feature selectors. Area, MOMent4, Skewness, Kurtosis, and Norm value could be marked as the best features in the breast images with 3 degrees of 0, 45, and 90 before ice test and Energy, Perimeter, Kurtosis, and Hurst coefficient could be marked as the best features in the breast images with 3 degrees of 0,45 , and 90 after ice test. The selected features were evaluated by different classifiers. Finally, to evaluate the proposed algorithm, 20-fold cross validation for left breast images and 22-fold cross validation for right breast images were used. The best combination obtained before ice test for right breast images was of AdaBoost and GA for the combination of 3 degrees of 0,45 , and 90 with mean accuracy of $88.03 \%$; and, for left breast images, it was again of AdaBoost and GA for 0 degree with mean accuracy of $85.33 \%$. The best combination obtained after ice test for right breast images was of AdaBoost and mRMR in 0 degree with mean accuracy of $88.03 \%$; and, for left breast images, it was of GA and AdaBoost for the combination of 3 degrees of 0,45 , and 90 with mean accuracy of $85 \%$. These combinations attained the maximum accuracy near $100 \%$. Also, FPRs for the mentioned combina- 
Table 4. Comparison of the proposed method and the previous methods.

\begin{tabular}{|c|c|c|c|c|c|c|c|c|}
\hline \multicolumn{4}{|c|}{ Methods } & $\begin{array}{c}\text { Mean } \\
\text { accuracy }\end{array}$ & $\begin{array}{c}\text { Max } \\
\text { accuracy }\end{array}$ & $\begin{array}{c}\text { Max } \\
\text { sensitivity }\end{array}$ & $\begin{array}{c}\text { Max } \\
\text { specificity }\end{array}$ & $\begin{array}{l}\text { Max } \\
\text { FPR }\end{array}$ \\
\hline \multicolumn{4}{|l|}{ Lee and Yang [37] } & - & - & - & - & $8.6 \%$ \\
\hline \multicolumn{4}{|l|}{ Yaneli et al. [38] } & - & $78.5 \%$ & - & - & - \\
\hline \multicolumn{4}{|c|}{ Dinsha and Manikandaprabu [22] } & - & $92.86 \%$ & $92.93 \%$ & - & - \\
\hline \multicolumn{4}{|l|}{ Araujo et al. [39] } & - & $84 \%$ & $85.7 \%$ & $86.5 \%$ & $16 \%$ \\
\hline \multirow{2}{*}{\multicolumn{2}{|c|}{ Lashkari et al. [25] }} & Right & $0^{\circ}$ & $87.42 \%$ & $100 \%$ & $100 \%$ & $100 \%$ & 0 \\
\hline & & Left & $0^{\circ}$ & $85.33 \%$ & $100 \%$ & $100 \%$ & $100 \%$ & 0 \\
\hline \multirow[b]{2}{*}{ Proposed method } & \multirow{2}{*}{$\mathrm{BIC}$} & Right & $0^{\circ} \& 45^{\circ} \& 90^{\circ}$ & $88.03 \%$ & $100 \%$ & $100 \%$ & $100 \%$ & 0 \\
\hline & & Left & $0^{\circ}$ & $85.33 \%$ & $100 \%$ & $100 \%$ & $100 \%$ & 0 \\
\hline \multirow[b]{2}{*}{ Proposed method } & \multirow{2}{*}{$\mathrm{AIC}^{\mathrm{b}}$} & Right & $0^{\circ}$ & $88.03 \%$ & $100 \%$ & $100 \%$ & $100 \%$ & 0 \\
\hline & & Left & $0^{\circ} \& 45^{\circ} \& 90^{\circ}$ & $85 \%$ & $100 \%$ & $100 \%$ & $100 \%$ & 0 \\
\hline
\end{tabular}

${ }^{\mathrm{a}}$ BIC: Before Ice Test; ${ }^{\mathrm{b}} \mathrm{AIC}$ : After Ice Test.

tions were equal to $0.0748,0.0917,0.0748$, and 0.0938 , respectively. The minimum FPR in these combinations was reduced to near zero. It should be noted that the important reasons for the difference in results among different degrees of breast images are the challenges in existing the mass or lesion in breast and unstable environment; considering the existing problems in ice test and little differences in the obtained accuracy in different situations, the cold stimulation can be omitted. But, the temperature diagram and thermal changes of each person can be used for detecting the presence or absence of masses. Thus, plotting the temperature diagram can be a way to help early detection, not necessarily to increase accuracy. Moreover, one of the reasons to justify the difference between the results for left and right breasts can be in equality in $\mathrm{TH}_{1}-\mathrm{TH}_{5}$. Table 4 shows the comparison between results of the proposed method and other methods. The obtained results indicate high efficiency of the presented algorithm. The best accuracy of the mentioned methods in this table compared with the best accuracy of the proposed method is notable [37-39].

\section{Acknowledgments}

The authors would like to thank 'Fanavaran Madoon Ghermez (FMG)' Co., Ltd and Mr. Mansoor Alidoosti and Ms Mitra Navid for their kind help and cooperation through their technical supports and acquiring data during working on this project. Also, all the patients who participated in this study are appreciated. This project is registered and funded by 'Iranian Research Organization for Science \& Technology (IROST) \&
Iranian National Science Foundation (INSF)' by registration number: 92000118.

\section{References}

1. Liberman, L., Abramson, A.F., Squires, F.B., Glassman, J.R., Morris, E.A., and Dershaw, D.D. "The breast imaging reporting and data system: positive predictive value of mammographic features and final assessment categories", AJR. Am. J. Roentgenol, 171(1), pp. 35-40 (1998).

2. Liberman, L. "Clinical management issues in percutaneous core breast biopsy", Radiol. Clin. North. Am., 38(4), pp. 791-807 (2000).

3. Rane, K.P., Joshi, R.R., and Chaudhari, L.A. "IR Imaging: A new approach for breast cancer detection", IJARECE (2014). Available from: technicheedu.in/journals/index.php/ijaecs/article/view/104

4. Zhang, Y.-D., Wang, S.-H., Liu, G., and Yang, J. "Computer-aided diagnosis of abnormal breasts in mammogram images by weighted-type fractional Fourier transform", Advances in Mechanical Engineering, 8(2), pp. 1-11 (2016).

5. Arena, F., Barone, C., and DiCicco, T. "Use of digital infrared imaging in enhanced breast cancer detection and monitoring of the clinical response to treatment", Proceedings of the 25th Annual International Conference of the IEEE, New York, NY, USA, pp. 1129-1132 (2003).

6. Gautherie, M., Atlas of Breast Thermography with Specific Guidelines for Examination and Interpretation, PAPUSA, Milan, Italy (1989).

7. Ng, E.Y., Ung, L.N., Ng, F.C., and Sim, L.S. "Statistical analysis of healthy and malignant breast 
thermography", J. Med. Eng. Technol., 25(6), pp. 253263 (2001).

8. Amalu, W.C., Hobbins, W.B., Head, J.F., and Elliot, R.L. Infrared Imaging of the Breast-An Overview, The Biomedical Engineering Handbook, 3rd Ed., Medical Devices and Systems. CRC Press, Baton Rouge (2006).

9. Ghayoumi Zadeh, H., Haddadnia, J., Hashemian, M., and Hassanpour, K. "Diagnosis of breast cancer using a combination of genetic algorithm and artificial neural network in medical infrared thermal imaging", Iran $J$. Med. Phys., 9(4), pp. 265-274 (2012).

10. Zhang, H., Li, K.Y., Sun, S.R., Wan, Y.W., Yao, X.L., and Zhang, X.L. "The Value-exploration of the clinical breast diagnosis by using thermal tomography", Fourth International Conference on Natural Computation, Jinan, China, pp. 138-142 (2008).

11. Williams, K.L., Williams, F.J., and Handley, R.S. "Infra-red thermometry in the diagnosis of breast disease", Lancet, 2(7217), pp. 1378-1381 (1961).

12. Parisky, Y.R., Sardi, A., Hamm, R., Hughes, K., Esserman, L., and Rust, S. "Efficacy of computerized infrared imaging analysis to evaluate mammographically suspicious lesions", AJR. Am. J. Roentgenol, 180(1), pp. 263-269 (2003).

13. Arora, N., Martins, D., Ruggerio, D., Tousimis, E., Swistel, A.J., and Osborne, M.P. "Effectiveness of a noninvasive digital infrared thermal imaging system in the detection of breast cancer", Am. J. Surg., 196(4), pp. 523-526 (2008).

14. Kennedy, D.A., Lee, T., and Seely, D. "A comparative review of thermography as a breast cancer screening technique", Integr. Cancer. Ther., 8(1), pp. 9-16 (2009).

15. Tang, X., Ding, H., Yuan, Y., and Wang, Q. "Morphological measurement of localized temperature increase amplitudes in breast infrared thermograms and its clinical application", Biomed Signal Process Control, 3(4), pp. 312-318 (2008).

16. Schaefer, G., Zavisek, M., and Nakashima, T. "Thermography based breast cancer analysis using statistical features and fuzzy classification", Pattern Recogn., 42(6), pp. 1133-1137 (2009).

17. Acharya, U.R., Ng, E.Y., Tan, J.H., and Sree, S.V. "Thermography based breast cancer detection using texture features and support vector machine", J. Med. Syst., 36(3), pp. 1503-1510 (2012).

18. Satoto, K.I., Nurhayati, O.D., and Isnanto, R.R. "Pattern recognition to detect breast cancer thermogram images based on fuzzy inference system method", IJCST, 2(3), pp. 484-487 (2011).

19. Kapoor, P., Prasad, S.V.A.V., and Patni, S. "Automatic analysis of breast tomograms for tumor detection based on biostatistical feature extraction and ANN", IJETED, 7(2), pp. 245-255 (2012).

20. Kapoor, P., Prasad, S.V.A.V., and Patni, S. "Image segmentation and asymmetry analysis of breast thermograms for tumor detection", IJCA, 50(9), pp. 40-45 (2012).
21. Nicandro, C.R., Efren, M.M., Yaneli, A.A.M., Enrique, M.D.C.M., Gabriel, A.M.H., and Nancy, P.C. "Evaluation of the diagnostic power of thermography in breast cancer using Bayesian network classifiers", Comput. Math. Methods Med., Article ID 264246, p. 10 (2013).

22. Dinsha, D. and Manikandaprabu, N. "Breast tumor segmentation and classification using SVM and Bayesian from thermogram images", Unique J. Eng. Adv. Sci., 2(2), pp. 147-151 (2014).

23. Fanavaran Madoon Ghermez (FMG) Co., Ltd [Internet]. Available from: http://www.ir-tc.com (Accessed Date: April 2016).

24. Imam Khomeini Hospital Official Website [Internet]. Available from: http://ikhc.tums.ac.ir/en (Accessed Date: April 2016).

25. Lashkari, A.E., Pak, F., and Firouzmand, M. "Full intelligent cancer classification of thermal breast images to assist physician in clinical diagnostic applications", J. Med. Signals Sens., 6(1), pp. 12-24 (2016).

26. Expanding the use of thermal imaging in the diagnosis of breast cancer. Documents 002-005, Ver 01. Available from: www.fmg-med.ir (Accessed Date: April 2016).

27. Gautherie, M., Haehnel, P., Walter, J.P., and Keith, L.G. "Thermovascular changes associated with in situ and minimal breast cancers. Results of an ongoing prospective study after four years", J. Reprod. Med., 32(11), pp. 833-842 (1987).

28. Peng, H., Long, F. and Ding, C. "Feature selection based on mutual information: criteria of maxdependency, max-relevance, and min-redundancy", IEEE Trans Pattern Anal Mach Intell, 27(8), pp. 1226-1238 (2005).

29. Whitney, A.W. "A direct method of nonparametric measurement selection", IEEE Transaction on Computers, 20(9), pp. 1100-1103 (1971).

30. Pudil, P., Novovicova, J., and Kittler, J. "Floating search methods in feature-selection", Pattern Recogn. Lett., 15(11), pp. 1119-1125 (1994).

31. Martin-Bautista, M.J. and Vila, M.A. "A survey of genetic feature selection in mining issues", 2, pp. 13141321 (1999).

32. Freund, Y. and Schapire, R.E. "A decision-theoretic generalization of on-line learning and an application to boosting", Journal of Computer and System Sciences, 55(1), pp. 119-139 (1997).

33. Schapire, R.E. and Singer, Y. "Improved boosting algorithms using confidence-rated predictions", Mach. Learn., 37(3), pp. 297-336 (1999).

34. Burges, C.J.C. "A tutorial on support vector machines for pattern recognition", Data Mining Knowl. Discov., 2, pp. 121-167 (1998).

35. Mitchell, T.M., Machine Learning, Burr Ridge, IL: McGraw Hill (1997).

36. Specht, D.F. "Probabilistic neural network for classification, map, or associative memory", In Proc. IEEE Int Conf Neural Netw, 1, pp. 525-532 (1988). 
37. Lee, M.Y. and Yang, C.S. "Entropy-based feature extraction and decision tree induction for breast cancer diagnosis with standardized thermograph images", Computer Methods and Programs in Biomedicine, 100(3), pp. 169-282 (2010).

38. Yaneli, A.A.M., Nicandro, C.R., Efren, M.M., Enrique, M.D.C.M., Nancy, P.C. and Gabriel, A.M.H. "Assessment of Bayesian network classifiers as tools for discriminating breast cancer pre-diagnosis based on three diagnostic methods", Lecture Notes in Computer Science, 7629, pp. 419-431 (2012).

39. Araujo, M.C., Lima, R.C.F., and de Souza, R.M.C.R. "Interval symbolic feature extraction for thermography breast cancer detection", Expert Syst. Appl., 14(15), pp. 6728-6737 (2014).

\section{Biographies}

Amir Ehsan Lashkari received the BSc degree in Biomedical Engineering from University of Isfahan, Isfahan, Iran, in 2009. He received the MSc degree in Biomedical Engineering from the Institute of Electrical and Computer Engineering at University of Tehran, Tehran, Iran, in 2011. He also received $\mathrm{PhD}$ degree in Biomedical Engineering from the Electrical and Information Technology Institute at Iranian Research Organization for Science and Technology (IROST), Tehran, Iran, in 2016. His research interests include digital image processing, neural networks, pattern recognition, intelligent systems, machine vision, fuzzy logic, chaos and fractal phenomena, digital design, artificial intelligent, digital signal processing, network and path planning, multiple valued function, and programmable devices. He has published more than 10 papers in these fields up to now.

Mohammad Firouzmand received his MSc degree in Medical Engineering from Sharif University of Technology in 1992 and his PhD degree in Signal Processing from the Institute National Poly-technique de Grenoble (INPG), France, in 2007. His research interests include bio-instruments, signal processing, medical image, and audio signal processing. He has published several research papers in these areas. 